\title{
Successful Treatment of Advanced Thymic Carcinoma with Carboplatin plus nab- Paclitaxel and Maintenance Monotherapy with nab-Paclitaxel: Two Case Reports
}

\author{
Tomoyuki Araya ${ }^{a}$ Toshiyuki Kita ${ }^{a}$ Hiroki Matsuoka ${ }^{a}$ Tamami Sakai ${ }^{a}$ \\ ${\text { Hideharu Kimurab }{ }^{b} \text { Kazuo Kasaharab }}^{b}$ \\ aDepartment of Respiratory Medicine, National Hospital Organization Kanazawa Medical \\ Center, Kanazawa, Japan; bepartment of Respiratory Medicine, Kanazawa University \\ Hospital, Kanazawa, Japan
}

Keywords

Thymic carcinoma $\cdot$ nab-paclitaxel $\cdot$ Carboplatin $\cdot$ Maintenance

\begin{abstract}
A standard chemotherapy regimen for advanced thymic carcinoma has not yet been established. We treated 2 cases of thymic carcinoma with carboplatin plus nanoparticle albuminbound (nab)-paclitaxel, and nab-paclitaxel maintenance therapy. The first case was a 68-yearold female, admitted for dyspnea and left shoulder pain. Chest computed tomography (CT) showed a huge mass in the anterior mediastinum, pleural and pericardial effusions, and multiple lung metastases. Specimens obtained from the anterior mediastinal mass by CT-guided needle biopsy revealed squamous cell carcinoma of the thymus, which was in stage IVB. The patient was administered carboplatin plus nab-paclitaxel as first-line treatment. After 3 cycles of chemotherapy, a partial response was observed with marked shrinkage of the tumor. Following 6 cycles of chemotherapy, nab-paclitaxel maintenance therapy was initiated. Disease progression was seen 9.1 months after initiation of treatment. The patient experienced no serious adverse events. The second case was a 70-year-old male who had productive cough, dyspnea, and right-sided chest pain. Chest $\mathrm{CT}$ revealed a huge mass in the anterior mediastinum, pericardial effusion, and multiple lymphadenopathies. Specimens obtained from station $11 \mathrm{~s}$ by endobronchial ultrasound-guided transbronchial needle aspiration revealed undifferentiated thymic carcinoma, which was in stage IVB. Six cycles of carboplatin plus nab-paclitaxel were administered, followed by 5 cycles of nab-paclitaxel for maintenance. A partial response was seen, which was sustained for more than 13 months. The patient experienced no serious adverse events. These cases show that chemotherapy with carboplatin plus nab-paclitaxel and $n a b$-paclitaxel as maintenance therapy can be a useful regimen for advanced thymic carcinoma.
\end{abstract}




\section{Introduction}

Thymic carcinoma is a rare malignant tumor that arises from the thymic epithelium in the anterior mediastinum, and has been reported to account for $12-14 \%$ of all thymic epithelial tumors, with a poor outcome due to its aggressive nature [1, 2]. Systemic chemotherapy is often administered in advanced cases. A standard chemotherapy regimen for thymic carcinoma has not yet been established due to its rarity, although a combination chemotherapy with carboplatin plus solvent-based paclitaxel has been reported to be effective for advanced thymic carcinoma [3-7]. However, little is known about the efficacy and safety of carboplatin plus nanoparticle albumin-bound (nab)-paclitaxel for advanced thymic carcinoma.

We report 2 cases of advanced thymic carcinoma successfully treated with carboplatin plus nab-paclitaxel and maintenance therapy with $n a b$-paclitaxel.

\section{Case Presentation}

Case 1

A 68-year-old female with no history of smoking was admitted to our hospital in January 2019 for dyspnea, left shoulder pain, and a widened mediastinum visible on chest X-ray. Chest computed tomography (CT) showed a huge mass in the anterior mediastinum, right hilar lymphadenopathy, massive left-sided thoracic effusion, pericardial effusion, and multiple lung nodules (Fig. 1a). Positron emission tomography revealed marked ${ }^{18}$ F-fluorodeoxyglucose accumulation in the anterior mediastinal mass, right hilum, left pleura, pericardium, bilateral lung nodules, and sternum. The specimens obtained from the anterior mediastinal mass by percutaneous CT-guided needle biopsy revealed squamous cell carcinoma of the thymus.

Based on these findings, the patient was diagnosed with stage IVB (cT3N3M1b) thymic carcinoma according to the Masaoka classification [8]. The level of serum CYFRA 21-1 was slightly elevated $(4.6 \mathrm{ng} / \mathrm{mL}$; normal range $\leq 3.5$ ). After drainage of the malignant effusion in the left pleura and pleurodesis, the patient had an Eastern Cooperative Oncology Group performance status (ECOG PS) of 2. The patient received chemotherapy with carboplatin plus $n a b$-paclitaxel as the first-line treatment, based on a previous study on non-small cell lung cancer (NSCLC) [9]. Carboplatin was administered on day 1 at a dose of targeted area under
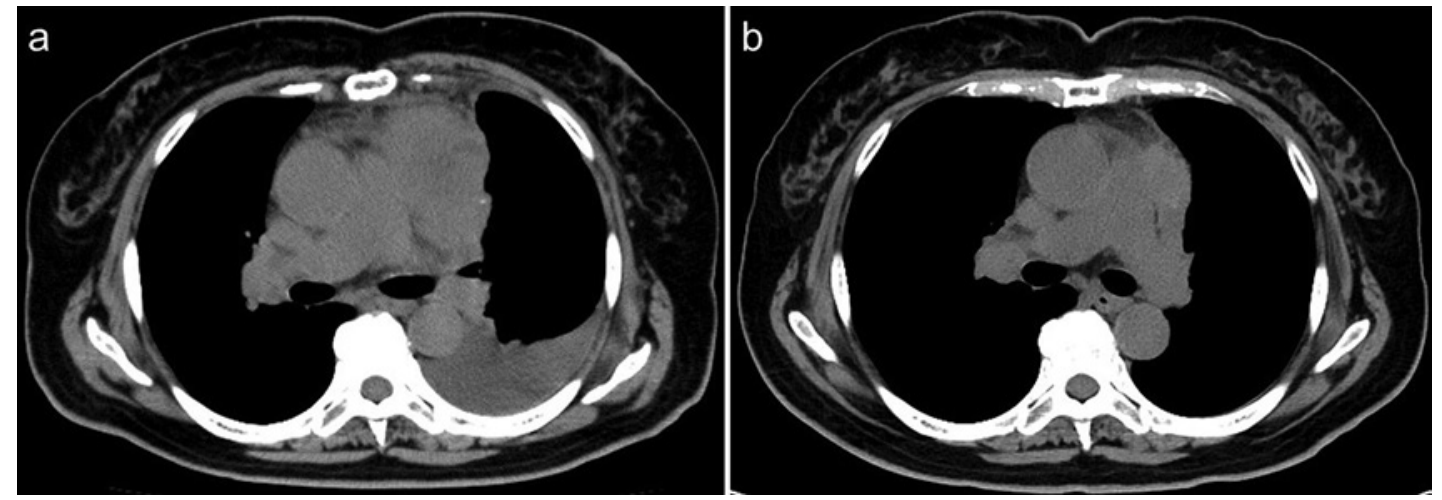

Fig. 1. Chest computed tomography scans at the time of admission showed a huge mass in the anterior mediastinum and left pleural effusion (a). A partial response was observed, with marked shrinkage of the anterior mediastinal tumor and disappearance of effusions after 3 cycles of combination chemotherapy (b). 



Fig. 2. Chest computed tomography scans at the time of admission showed a huge mass in the anterior mediastinum (a) and lymphadenopathy of the right hilar and mediastinal nodes (b). After 4 cycles of combination chemotherapy, a partial response was observed, with marked shrinkage of the anterior mediastinal tumor (c) and lymphadenopathy (c, d).

the concentration-time curve of 6 and nab-paclitaxel at a dose of $100 \mathrm{mg} / \mathrm{m}^{2}$ on days 1,8 , and 15; this combination was repeated every 4 weeks. After 3 cycles of combination chemotherapy, a partial response was observed with marked shrinkage of the anterior mediastinal tumor and absence of effusions (Fig. 1b).

At this point, the ECOG PS of the patient had improved to 0 . The serum level of CYFRA 21-1 had returned to normal limits. Following 6 cycles of combination chemotherapy, maintenance therapy with $n a b$-paclitaxel at a dose of $100 \mathrm{mg} / \mathrm{m}^{2}$ on days 1,8 , and 15 every 4 weeks was initiated, in accordance with the schedule of combination chemotherapy. Due to grade 3 neutropenia during the 4th, 5th, and 6th cycle of combination treatment, a total of 4 doses of $n a b$-paclitaxel on day 8 or 15 were omitted. Neither a dose delay nor a reduction was required. During maintenance therapy, no modifications including dose interruption, dose delay, and dose reduction were required. The patient experienced no serious adverse events. Disease progression was observed 9.1 months after initiation of the first-line treatment. The patient is still alive and receiving S-1 as the third-line therapy, after failure of pemetrexed as second-line treatment.

Case 2

A 70-year-old male who smoked 64.5 packs per year was referred to our hospital with the chief complaints of productive cough, dyspnea, and right-sided chest pain in June 2019. 
Chest X-ray and CT revealed a huge mass in the anterior mediastinum, pericardial effusion, and lymphadenopathy of the right supraclavicular, right hilar, and bilateral mediastinal nodes (Fig. 2a, b). Positron emission tomography revealed marked accumulation of ${ }^{18}$ F-fluorodeoxyglucose in all these lesions. The tumor specimens obtained from mediastinal lymph node station $11 \mathrm{~s}$ by endobronchial ultrasound-guided transbronchial needle aspiration revealed undifferentiated carcinoma of the thymus.

Based on these findings, the patient was diagnosed with stage IVB (cT4N2M1b) thymic carcinoma according to the Masaoka classification. The levels of serum squamous cell carcinoma antigen and neuron-specific enolase were elevated at $10.5 \mathrm{ng} / \mathrm{mL}$ (normal $\leq 1.5$ ) and $71.4 \mathrm{ng} / \mathrm{mL}$ (normal $\leq 16.3$ ), respectively. At the time of diagnosis, the patient had an ECOG PS of 2 . The patient received combination chemotherapy comprising carboplatin plus $n a b$-paclitaxel as first-line treatment. Carboplatin was administered on day 1 at a dose of targeted area under the concentration-time curve of 6 and $n a b$-paclitaxel at a dose of $100 \mathrm{mg} /$ $\mathrm{m}^{2}$ on days 1 and 8 , and this combination was repeated every 4 weeks. After 4 cycles of combination chemotherapy, a partial response was observed with marked shrinkage of the anterior mediastinal tumor and lymphadenopathy of the right hilar and bilateral mediastinal nodes (Fig. 2c, d).

At this point, the ECOG PS of the patient had improved to 0 . The serum levels of squamous cell carcinoma antigen and neuron-specific enolase had returned to normal limits. Due to grade 3 neutropenia or grade 3 thrombocytopenia during the 6 cycles of combination chemotherapy, a total of 7 doses of $n a b$-paclitaxel on day 8 or 15 were omitted. In addition, 1- to 2 -week dose delays per cycle were required during the combination chemotherapy. A dose reduction was not required. Therefore, after a total of 6 cycles of combination chemotherapy, maintenance therapy with $n a b$-paclitaxel at a dose of $100 \mathrm{mg} / \mathrm{m}^{2}$ on days 1 and 8 every 4 weeks was initiated in accordance with the schedule of prior combination therapy. During maintenance therapy, a week's delay per cycle was required. The patient experienced no serious adverse events. The patient has been receiving the first-line treatment for more than 13 months with no evidence of progression.

\section{Discussion}

The present case study suggests that combination chemotherapy comprising carboplatin plus nab-paclitaxel and maintenance therapy with $n a b$-paclitaxel is a promising treatment for advanced thymic carcinoma. In addition, both our patients had an ECOG PS of 2 at the initiation of combination chemotherapy; therefore, this combination therapy and maintenance therapy with $n a b$-paclitaxel is feasible for patients with a poor PS and advanced thymic carcinoma. Furthermore, this is the first report to demonstrate that combination chemotherapy comprising carboplatin plus nab-paclitaxel and maintenance therapy with $n a b$-paclitaxel is effective for undifferentiated thymic carcinoma, a rare type of thymic carcinoma, although previous reports had showed the efficacy of this treatment in other subtypes of thymic carcinoma [10-13]. Several retrospective [3,4] and prospective [5-7] studies have reported that carboplatin plus solvent-based paclitaxel is an effective treatment for thymic cancer. Median progression-free survival (PFS) was 5.0-8.6 months with the regimen comprising carboplatin plus solvent-based paclitaxel [3-7]. However, this combination can cause severe peripheral neuropathy, which might require dose reductions and/or discontinuation of treatment. Recently, it has been reported that nab-paclitaxel combined with carboplatin showed higher efficacy and less toxicity such as neuropathy, neutropenia, myalgia, and arthralgia in the treatment of NSCLC compared to carboplatin plus solvent-based paclitaxel [9]. In addition, maintenance monotherapy with nab-paclitaxel following combination

\section{Karger'}




\section{Case Reports in Oncology}

Case Rep Oncol 2020;13:1506-1512 DOI: $10.1159 / 000510894$

(c) 2020 The Author(s). Published by S. Karger AG, Basel www.karger.com/cro

Araya et al.: Carboplatin + nab-Paclitaxel and $n a b$-Paclitaxel Maintenance for Thymic Carcinoma



\section{Karger'}


chemotherapy with carboplatin plus nab-paclitaxel is reported to be a useful treatment option for patients with advanced NSCLC [14]. Based on these findings, our patients received the same regimen. The PFS among our patients was longer than that among patients treated with carboplatin plus solvent-based paclitaxel. Table 1 summarizes the results of previously published reports evaluating the efficacy and safety of this combination chemotherapy for advanced thymic carcinoma. Of these reports, only one report described the period of PFS and overall survival. Therefore, little is known about the exact efficacy of carboplatin plus nabpaclitaxel for advanced thymic carcinoma.

With respect to adverse events, in these 2 cases, several doses of $n a b$-paclitaxel were omitted due to hematological toxicities. Nevertheless, this chemotherapy regimen was effective. One of the reasons for the good results is that the dose of $n a b$-paclitaxel in this regimen is easy to adjust according to the adverse events observed. Therefore, we believe that combination chemotherapy with carboplatin plus nab-paclitaxel and continuation maintenance with $n a b$-paclitaxel is the best alternative for patients with advanced thymic carcinoma and a poor PS.

\section{Conclusions}

Combination chemotherapy with carboplatin plus nab-paclitaxel and continuation maintenance with $n a b$-paclitaxel can be a useful regimen for advanced thymic carcinoma. A future prospective study is necessary to evaluate the efficacy and safety of this regimen for advanced thymic carcinoma.

\section{Acknowledgements}

We would like to thank Editage (https://www.editage.jp/) for English language editing.

\section{Statement of Ethics}

The case study was approved by the ethics committees of the National Hospital Organization Kanazawa Medical Center (\#R02-034). Written informed consent was obtained from the patients for publication of this case report and any accompanying images. The case study was conducted ethically in accordance with the World Medical Association Declaration of Helsinki.

\section{Conflict of Interest Statement}

The authors have no conflicts of interest to declare.

\section{Funding Sources}

The present study did not require external funding support. 


\section{Case Reports in Oncology}

\begin{tabular}{l|l}
\hline Case Rep Oncol 2020;13:1506-1512 \\
\hline DOI: 10.1159/000510894 & $\begin{array}{l}\text { @ 2020 The Author(s). Published by S. Karger AG, Basel } \\
\text { www.karger.com/cro }\end{array}$ \\
\hline
\end{tabular}

Araya et al.: Carboplatin + nab-Paclitaxel and nab-Paclitaxel Maintenance for Thymic Carcinoma

\section{Author Contributions}

Each author contributed to the study as follows: conception and design of the work - T.A., T.K.; data acquisition - T.A., T.K.; drafting the work or revising it critically - T.A., T.K., H.M., T.S., H.K., and K.K.; T.K. is the guarantor of the paper, taking responsibility for the integrity of the work as a whole, from inception to publication.

\section{References}

1 Kondo K, Monden Y. Therapy for thymic epithelial tumors: a clinical study of 1,320 patients from Japan. Ann Thorac Surg. 2003 Sep; 76(3):878-84; discussion 884-5.

2 Nakajima J, Okumura M, Yano M, Date H, Onuki T, Haniuda M, et al. Myasthenia gravis with thymic epithelial tumour: a retrospective analysis of a Japanese database. Eur J Cardiothorac Surg. 2016 May;49(5):1510-5.

3 Igawa S, Murakami H, Takahashi T, Nakamura Y, Tsuya A, Naito T, et al. Efficacy of chemotherapy with carboplatin and paclitaxel for unresectable thymic carcinoma. Lung Cancer. 2010 Feb;67(2):194-7.

4 Furugen M, Sekine I, Tsuta K, Horinouchi H, Nokihara H, Yamamoto N, et al. Combination chemotherapy with carboplatin and paclitaxel for advanced thymic cancer. Jpn J Clin Oncol. 2011 Aug;41(8):1013-6.

5 Lemma GL, Lee JW, Aisner SC, Langer CJ, Tester WJ, Johnson DH, et al. Phase II study of carboplatin and paclitaxel in advanced thymoma and thymic carcinoma. J Clin Oncol. 2011 May;29(15):2060-5.

6 Hirai F, Yamanaka T, Taguchi K, Daga H, Ono A, Tanaka K, et al. A multicenter phase II study of carboplatin and paclitaxel for advanced thymic carcinoma: WJOG4207L. Ann Oncol. 2015 Feb;26(2):363-8.

7 Xu JP, Hao XZ, Zhang XR, Yang S, Shi YK. Efficacy and safety of the combination of paclitaxel and platinum in advanced thymic carcinoma. Thorac Cancer. 2016 Mar; 7(2):222-5.

8 Masaoka A, Monden Y, Nakahara K, Tanioka T. Follow-up study of thymomas with special reference to their clinical stages. Cancer. 1981 Dec;48(11):2485-92.

9 Socinski MA, Bondarenko I, Karaseva NA, Makhson AM, Vynnychenko I, Okamoto I, et al. Weekly nab-paclitaxel in combination with carboplatin versus solvent-based paclitaxel plus carboplatin as first-line therapy in patients with advanced non-small-cell lung cancer: final results of a phase III trial. J Clin Oncol. 2012 Jun; 30(17):2055-62.

10 Makimoto G, Fujiwara K, Watanabe H, Kameyama N, Matsushita M, Rai K, et al. nab-paclitaxel in combination with carboplatin for a previously treated thymic carcinoma. Case Rep Oncol. 2014 Jan;7(1):14-7.

11 Igawa S, Yanagisawa N, Niwa H, Ishihara M, Hiyoshi Y, Otani S, et al. Successful chemotherapy with carboplatin and nab-paclitaxel for thymic large cell neuroendocrine carcinoma: a case report. Oncol Lett. 2015 Dec;10(6): 3519-22.

12 Shima H, Ozasa H, Tsuji T, Ajimizu H, Nomizo T, Yagi Y, et al. Response to chemotherapy with carboplatin plus albumin-bound paclitaxel in a patient with lymphoepithelioma-like thymic carcinoma: a case report. Mol Clin Oncol. 2016 May;4(5):715-8.

13 Funaishi K, Yamasaki M, Saito N, Daido W, Ishiyama S, Deguchi N, et al. First-line treatment with carboplatin plus nab-paclitaxel and maintenance monotherapy with nab-paclitaxel for a thymic carcinoma: a case report. Case Rep Oncol. 2017 May-Aug;10(2):571-6.

14 Nakao A, Uchino J, Igata F, On R, Ikeda T, Yatsugi H, et al. nab-paclitaxel maintenance therapy following carboplatin + nab-paclitaxel combination therapy in chemotherapy naïve patients with advanced non-small cell lung cancer: multicenter, open-label, single-arm phase II trial. Invest New Drugs. 2018 Oct;36(5):903-10. 